Bio - grafia. Escritos sobre la Biología y su Enseñanza. ISSN 2027

Edición Extraordinaria. p.p. 344 - 354

Memorias del VIII Encuentro Nacional de Experiencias en Enseñanza de la Biología y la Educación Ambiental. III Congreso Nacional de Investigación en Enseñanza de la Biología.

\title{
TÍTULO: "Valoración de los Proyectos de Grado sobre Educación Ambiental en la Universidad, Aplicando la Hipótesis de Progresión".
}

Title: Evaluation of undergraduate research projects on Environmental Education at university, implementing the hypothesis of progression

DRA ALBA LEONILDE SUÁREZ ARIAS Universidad del Quindío.

DRA: MERCE JUNYENT PUBILL Universidad Autónoma de Barcelona, España

DR: JAVIER GARCÍA GÓMEZ Universidad de Valencia, España

\section{RESUMEN:}

Este trabajo aplica la hipótesis de progresión como metodología para la valoración de los proyectos de grado de los estudiantes en Educación Ambiental en la Universidad.

Se han analizado, los proyectos realizados por los alumnos de la licenciatura en Biología y Educación Ambiental, en la Universidad del Quindío, Colombia. Cuyo objetivo tiene la formación de profesores para los diferentes niveles educativos. La hipótesis de progresión permite valorar el cambio conceptual presentado en los proyectos de grado no como un proceso puntual sino como un proceso de evolución de los contenidos.

La investigación muestra que los Proyectos fin de grado de los estudiantes de Biología y educación Ambiental valorados aplicando la hipótesis de progresión tienen una visión aditiva y poco fundamentada sobre la incorpora ración de la educación ambiental en el currículo

\section{ABSTRACT}

This paper aims to explain the hypothesis of progression as a methodology for the assessment of research projects of undergraduate students in environmental education at the University. 
Bio - grafia. Escritos sobre la Biología y su Enseñanza. ISSN 2027

Edición Extraordinaria. p.p. 344 - 354

Memorias del VIII Encuentro Nacional de Experiencias en Enseñanza de la Biología y la Educación Ambiental. III Congreso Nacional de Investigación en Enseñanza de la Biología.

We analyzed the projects carried out by students of the degree in Biology and Environmental Education at the University of Quindío, Colombia. Whose goal is the training of teachers for different educational levels.

The hypothesis of progression allows to assess the conceptual change proposed in the research project, not as a punctual process but as a process of evolution of the contents.

Research shows that students' final degree projects of biology and environmental education evaluated using the hypothesis of progression have an additive view and little based about the incorporation of environmental education into the curriculum

PALABRAS CLAVES: educación ambiental, Hipótesis de progresión, investigación cualitativa, proyectos, universidad.

Key words: Environmental Education, Hypothesis of Progression, Qualitative Research, Projects, University.

\section{INTRODUCCIÓN}

La investigación es un aporte a la adecuación de la propuesta curricular, insertando en su contexto competencias en educación para la sostenibilidad como una necesidad de intervención en sus prácticas para promover conocimientos, actitudes, habilidades, valores y acciones que contribuyen a incrementar la participación de los alumnos ciudadanos y futuros profesionales en la construcción de sociedades sostenibles. También podría favorecer sus actuaciones como agentes de cambios en relación a las cuestiones sociales y ambientales. Lo anterior según la red ACES (formada por 11 universidades, 5 europeas y 6 latinoamericanas), por ejemplo, trabaja en el diseño de modelos, criterios e instrumentos de ambientalización de los estudios superiores, además de compartir y contrastar sus proyectos y trabajos de ambientalización (Geli y Arbat, 2005).

Uno de los objetivos de la investigación es presentar la Hipótesis de Progresión como una metodología cualitativa para valorar los Proyectos de Grado sobre Educación Ambiental en la Universidad. 
Bio - grafia. Escritos sobre la Biología y su Enseñanza. ISSN 2027

Edición Extraordinaria. p.p. 344 - 354

Memorias del VIII Encuentro Nacional de Experiencias en Enseñanza de la Biología y la Educación Ambiental. III Congreso Nacional de Investigación en Enseñanza de la Biología.

Para ello se han analizado, los proyectos que incorporan la dimensión ambiental (del año 2009 hasta el 2012), realizados por los alumnos de la licenciatura en Biología y Educación Ambiental de la Universidad del Quindío, Colombia. Cuyo objetivo tiene la formación de profesores de diferentes niveles educativos. La hipótesis de progresión permite valorar el cambio conceptual presentado en los proyectos de grado por los estudiantes no como un proceso puntual sino como un proceso de evolución de los contenidos.

Las conclusiones a que llegamos no son generalizables, pues se trata de un estudio de caso aplicando una investigación donde el paradigma es interpretativo, con un enfoque de análisis cualitativo. Pero puede constituir, en todo caso, una contribución a la exploración de las ideas que tienen los estudiantes en general, aunque en nuestro caso se ha aplicado a los de la licenciatura en Biología y educación Ambiental en sus proyectos de grado, sobre la inclusión de la dimensión ambiental en el currículo de la Facultad de Educación.

\section{METODOLOGÍA}

Como hemos mencionado el Paradigma de investigación es interpretativo, con un enfoque de análisis cualitativo y que incluye lo cuantitativo. Como señala Junyent (2002) un paradigma en investigación y evaluación educativa es interactiva, implantada a la práctica y en un contexto, los métodos no son pre ordenados, sino emergentes las construcciones individuales son extraídas y refinadas a través de la interpretación y estrategias cualitativas. En este mismo sentido Wittrock, (1986) dice que, lo fundamental no es qué instrumento es mejor sino cuál es el más apropiado para la cuestión que se estudia y cuál representará adecuadamente el segmento de la realidad estudiada en razón al tipo de problema formulado, lo mismo que al tipo de marco teórico y conceptual presentado. La investigación presentada complementa las metodologías de tipo cualitativo con un análisis cuantitativo de los datos a través del método descriptivo utilizando la Moda estadística.

En cuanto a la intencionalidad, los enfoques de corte cuantitativo se centran en la explicación y la predicación de una realidad considerada en sus aspectos más universales y 
Bio - grafia. Escritos sobre la Biología y su Enseñanza. ISSN 2027

Edición Extraordinaria. p.p. 344 - 354

Memorias del VIII Encuentro Nacional de Experiencias en Enseñanza de la Biología y la Educación Ambiental. III Congreso Nacional de Investigación en Enseñanza de la Biología.

vistas desde una perspectiva externa (objetiva). Mientras que los de orden cualitativo se centra en la comprensión de una realidad considerada desde sus aspectos particulares como fruto de un proceso histórico de construcción y vista a partir de la lógica y el sentir de sus protagonistas, es decir desde una perspectiva interna (subjetiva). La metodología de investigación cualitativa es entendida como las acciones de descubrimientos de nuevos conceptos, dentro de una perspectiva holística (Quintana, 2006).

Para la obtención de los datos, se ha utilizado un cuestionario con 14 items y una matriz de codificación de la información, que se ha aplicado a 92 proyectos realizados durante cuatro años por los estudiantes de licenciatura en Biología y educación Ambiental (BYEA) de la Universidad del Quindío, Colombia. De todos los proyectos sólo cuatro incluían algún aspecto relacionado con la educación ambiental y a estos se les aplicó el análisis mediante la hipótesis de progresión. La información de los proyectos permite evidenciar la formación recibida en Educación Ambiental a lo largo de su carrera. Puesto que se realiza al final de sus estudios permite conocer cuál es su capacitación al momento de incorporarse a su actividad profesional

Los contenidos de los proyectos de grado de los estudiantes seleccionados, se han agrupado por categorías y subcategorías de análisis, para evidenciar si estos proyectos reflejan el qué y el cómo incorporar la dimensión ambiental en el currículo, posibilidades y condiciones de incorporarla y el tratamiento de los problemas ambientales. Las categorías y subcategorías se confeccionaron atendiendo al marco conceptual de la educación ambiental.

Por ello la primera labor fue el establecimiento del sistema de categorías a aplicar en el análisis de los proyectos de fin de grado (PFG), bajo los cuales se valorararon los contenidos que en ellos aparecen. Es decir se define una escala de categorías y sub categorías que va desde un conocimiento simple hasta otro más complejo (cuadro 1).

Como hemos mencionado, la metodología utilizada es cualitativa, pero apoyada en lo cuantitativo y se inclinó por un procedimiento de técnicas cualitativas que describe la 
Bio - grafia. Escritos sobre la Biología y su Enseñanza. ISSN 2027

Edición Extraordinaria. p.p. 344 - 354

Memorias del VIII Encuentro Nacional de Experiencias en Enseñanza de la Biología y la Educación Ambiental. III Congreso Nacional de Investigación en Enseñanza de la Biología.

complejidad del problema y la interacción entre categorías, (Bardin, 2002). Este enfoque es importante para comprender el discurso expuesto en los proyectos a partir de su desarrollo y resultados, lo que posibilita una aproximación a la subjetividad interpretativa de la realidad.

Para el estudio, el sistema de categorías se construye a partir de: necesidad, sobre el cómo y qué incluir de la educación ambiental, las posibilidades o condiciones de incorporar la educación ambiental y el tratamiento de los problemas ambientales.

Se estima relevante que las categorías de análisis que se han considerado y trabajado en los proyectos de grado en general, no en cada uno de sus materiales por separado, pues así los significados quedan mejor contextualizados con el propósito de identificar el grado de integración de los contenidos sobre Educación Ambiental registrados en los proyectos de grado.

\begin{tabular}{|c|c|}
\hline CATEGORÍAS & SUBCATEGORÍAS \\
\hline \multirow{7}{*}{$\begin{array}{l}\text { A. Necesidad, sobre el } \\
\text { cómo y qué incluir de la } \\
\text { educación ambiental. }\end{array}$} & A.1.Necesidad de incorporar la educación ambiental \\
\hline & A.2.Cómo incorporar la educación ambiental \\
\hline & $\begin{array}{l}\text { A.3. Importancia de la inclusión de la educación } \\
\text { ambiental en términos del desarrollo sostenible. }\end{array}$ \\
\hline & $\begin{array}{l}\text { A.4.Conocimientos profesionales y docentes_al } \\
\text { diseñar y poner en práctica los contenidos. }\end{array}$ \\
\hline & $\begin{array}{l}\text { A.5.Características los contenidos para un } \\
\text { aprendizaje significativo en los estudiantes. }\end{array}$ \\
\hline & A.6.Las ideas que tienen los estudiantes \\
\hline & A.7. Actividades y recursos que se plantean. \\
\hline \multirow{5}{*}{$\begin{array}{l}\text { B. Las posibilidades } 0 \\
\text { condiciones de incorporar } \\
\text { la educación ambiental. }\end{array}$} & B.1.Importancia de incorporar la educación ambiental \\
\hline & B.2.Limitantes para incluir la educación ambiental \\
\hline & $\begin{array}{l}\text { B.3.Cambios requeridos para incluir la educación } \\
\text { ambiental. }\end{array}$ \\
\hline & $\begin{array}{l}\text { B.4. conocimientos necesarios para que se incluya la } \\
\text { educación ambiental. }\end{array}$ \\
\hline & $\begin{array}{l}\text { B.5. Aspectos de la formación profesional y docente } \\
\text { para hacer efectiva la inclusión de la educación }\end{array}$ \\
\hline
\end{tabular}


Bio - grafia. Escritos sobre la Biología y su Enseñanza. ISSN 2027

Edición Extraordinaria. p.p. 344 - 354

Memorias del VIII Encuentro Nacional de Experiencias en Enseñanza de la Biología y la Educación Ambiental. III Congreso Nacional de Investigación en Enseñanza de la Biología.

\begin{tabular}{|l|l|}
\hline & ambiental. \\
\hline \multirow{2}{*}{$\begin{array}{l}\text { C. Tratamiento de los } \\
\text { problemas ambientales. }\end{array}$} & $\begin{array}{l}\text { C.1. Características de los problemas ambientales que } \\
\text { se presentan a nivel Global y local. }\end{array}$ \\
\cline { 2 - 2 } & $\begin{array}{l}\text { C.2. Principios filosóficos, científicos y sociológicos } \\
\text { necesarios para abordar los problemas ambientales. }\end{array}$ \\
\hline
\end{tabular}

Cuadro 1: Sistema de categorías.

Aunque se trata de una metodología cualitativa hemos asignado valores numéricos para discriminar los resultados. Los diferentes valores por categoría y subcategoría se recogen en el cuadro 2 a los que se les asignó un valor numérico de 1, 3 y 5 respectivamente. Estos valores son crecientes para identificar cuando un valor es de menor a mayor complejidad.

El primer nivel (1), refleja la valoración del proyecto dentro de un valor no deseable, supone una visión dogmática del conocimiento.

El segundo nivel (3), se refiere a aquellos modelos de proyectos que hacen de puente entre el valor no deseable y el valor deseable: el proyecto que presenta un discurso técnico y activista, caracterizado por un discurso aditivo y utilización de una metodología didáctica transmisiva

Por último, el tercer nivel (5) se corresponde con lo que consideramos como el perfil del proyecto deseable: que presenta un discurso desde la investigación en la acción, lo que supone una visión relativa, evolutiva e integradora del conocimiento, con un discurso constructivista del aprendizaje.

\begin{tabular}{|l|l|l|l|}
\cline { 2 - 4 } \multicolumn{1}{c|}{} & \multicolumn{3}{c|}{ Valores Propuestos } \\
\hline \multicolumn{1}{|c|}{ Categorías } & $\begin{array}{c}\text { Nivel no } \\
\text { deseable } \\
1\end{array}$ & $\begin{array}{c}\text { Nivel } \\
\text { Intermedio } \\
\mathbf{3}\end{array}$ & $\begin{array}{c}\text { Nivel } \\
\text { Deseable } \\
\mathbf{5}\end{array}$ \\
\hline $\begin{array}{l}\text { Categoría A: Contenidos que } \\
\text { tiene el proyecto sobre el } \\
\text { cómo y qué incluir de la } \\
\text { educación ambiental. }\end{array}$ & $\begin{array}{l}\text { Argumentos } \\
\text { escasos e } \\
\text { incoherencias } \\
\text { en la forma de }\end{array}$ & $\begin{array}{l}\text { Presenta un } \\
\text { reconocimiento } \\
\text { del cómo y qué } \\
\text { de la educación }\end{array}$ & $\begin{array}{l}\text { Proyectos } \\
\text { que denotan } \\
\text { una visión del } \\
\text { discurso }\end{array}$ \\
\hline
\end{tabular}


Bio - grafia. Escritos sobre la Biología y su Enseñanza. ISSN 2027

Edición Extraordinaria. p.p. 344 - 354

Memorias del VIII Encuentro Nacional de Experiencias en Enseñanza de la Biología y la Educación Ambiental. III Congreso Nacional de Investigación en Enseñanza de la Biología.

\begin{tabular}{|c|c|c|c|}
\hline $\begin{array}{l}\text { Categoría B: Contenidos que } \\
\text { tiene el proyecto sobre las } \\
\text { posibilidades ó condiciones de } \\
\text { incorporar la educación } \\
\text { ambiental. } \\
\text { Categoría C: Contenidos que } \\
\text { tiene el proyecto relacionadas } \\
\text { con el tratamiento de los } \\
\text { problemas ambientales. }\end{array}$ & $\begin{array}{l}\text { presentar el } \\
\text { discurso en el } \\
\text { proyecto. } \\
\text { No se } \\
\text { encuentra } \\
\text { dentro de valor } \\
\text { deseable } \\
\text { propuesto }\end{array}$ & $\begin{array}{l}\text { ambiental pero } \\
\text { se presenta } \\
\text { incompleta. } \\
\text { Con una, visión } \\
\text { aditiva, no se } \\
\text { integran las } \\
\text { partes ni se } \\
\text { justifican las } \\
\text { relaciones }\end{array}$ & $\begin{array}{l}\text { integral y } \\
\text { se presenta de } \\
\text { una } \\
\text { manera } \\
\text { fundamentada } \\
\text { Según el valor } \\
\text { deseable } \\
\text { propuesto. }\end{array}$ \\
\hline
\end{tabular}

Cuadro 2: Categorías y valores.

\section{RESULTADOS Y DISCUSIÓN DE LOS DATOS.}

La comparación entre los proyectos de grado de la licenciatura en Biología y Educación Ambiental con respecto a cada una de las categorías $A, B$ y $C$, (cuadro 2) se ha hecho con base a la Moda de cada una de las subcategorías en cada uno de los proyectos seleccionados. De la misma forma se analizaron los valores asignados para identificar los contenidos planteados en los proyectos, por ello se ha utilizado la Moda como parámetro para el análisis.

Categoría A: Necesidad sobre el cómo y el qué, incorporar la Educacación ambiental (IDA).

\begin{tabular}{|c|c|c|c|c|c|c|c|c|c|}
\cline { 3 - 9 } \multicolumn{2}{c|}{} & \multicolumn{7}{c|}{ Subcategorías } \\
\hline Proyectos & Años & $\begin{array}{c}\text { A. } \\
1 .\end{array}$ & A.2. & A.3. & A.4. & A.5. & A.6. & A.7 & $\begin{array}{c}\text { Valor de la } \\
\text { Moda } \\
\text { Por subcategoría }\end{array}$ \\
\hline P.36 & (2009) & 3 & 3 & 1 & 3 & 3 & 3 & 3 & 3 \\
\hline P.15 & $(2010)$ & 1 & 5 & 1 & 1 & 3 & 3 & 3 & 1 y 3* \\
\hline
\end{tabular}


Bio - grafia. Escritos sobre la Biología y su Enseñanza. ISSN 2027

Edición Extraordinaria. p.p. 344 - 354

Memorias del VIII Encuentro Nacional de Experiencias en Enseñanza de la Biología y la Educación Ambiental. III Congreso Nacional de Investigación en Enseñanza de la Biología.

\begin{tabular}{|c|c|c|c|c|c|c|c|c|c|}
\hline P.6. & $(2011)$ & 1 & 1 & 1 & 3 & 3 & 1 & 3 & 1 y 3* \\
\hline P.7. & (2012) & 5 & 5 & 1 & 5 & 5 & 5 & 5 & 5 \\
\hline $\begin{array}{c}\text { Valor de la Moda } \\
\text { por años }\end{array}$ & 1 & 5 & 1 & 3 & 3 & 3 & 3 & 3 \\
\hline
\end{tabular}

Cuadro 3: Proyectos de grado que consideran, el cómo y el qué, IEA.

A la vista de los resultados en el cuadro 3. En la categoría A: En los proyectos de grado de la licenciatura en BYEA, se observa que el valor predominante es el 3 (intermedio) para los años 2009, 2010 y 2011 o sea que los proyectos tienen una visión aditiva, no se integran sus partes ni se justifican sus relaciones y se hace poco fundamentado sobre el cómo y el qué IEA. Para los años 2010 y 2011 también se tienen como valores predominantes el 1 (valor no deseable), esto nos permite inferir que los proyectos presentan incoherencias sobre el cómo y el qué IEA. Para el año 2012 el valor predominante es el valor 5 (deseable) o sea que se tiene en el proyecto una visión articulada, integral y se hace fundamentada el cómo y el qué, IEA. Se muestra una situación diferente con los proyectos de los años 2009, 2010 y 2011, esto favorece la IEA. 
Bio - grafia. Escritos sobre la Biología y su Enseñanza. ISSN 2027

Edición Extraordinaria. p.p. 344 - 354

Memorias del VIII Encuentro Nacional de Experiencias en Enseñanza de la Biología y la Educación Ambiental. III Congreso Nacional de Investigación en Enseñanza de la Biología.

Categoría B: Sobre las posibilidades y condiciones de IEA.

\begin{tabular}{|c|c|c|c|c|c|c|c|}
\cline { 3 - 7 } \multicolumn{2}{c|}{} & \multicolumn{5}{c|}{ Subcategorías } & \multicolumn{1}{c|}{} \\
\hline Proyectos & Años & B.1. & B.2 & B.3 & B.4 & B.5 & $\begin{array}{c}\text { Valor de la Moda } \\
\text { por subcategoría }\end{array}$ \\
\hline P.36. & (2009) & 3 & 3 & 3 & 3 & 3 & 3 \\
\hline P.15. & $(2010)$ & 3 & 1 & 3 & 5 & 3 & 3 \\
\hline P.6. & $(2011)$ & 1 & 1 & 1 & 1 & 3 & $1^{\star}$ \\
\hline P.7. & (2012) & 5 & 5 & 5 & 5 & 5 & $5^{\star}$ \\
\hline & & 3 & 1 & 3 & 5 & 3 & 3 \\
\hline
\end{tabular}

Cuadro 4: Proyectos de grado que consideran, las posibilidades y condiciones de IEA.

En la categoría B, a la vista de los resultados según (cuadro 4). En los proyectos de grado de BYEA se observa que el valor predominante es el 3 (valor intermedio) para los años 2009 y 2010 o sea que los proyectos tienen

una visión aditiva, no se integran sus partes ni se justifican sus relaciones y es poco fundamentado, las posibilidades y condiciones de IDA. Para el año 2011 se muestra una situación diferente en relación a los años 2009, 2010 y 2012, ya que los proyectos de grado se ubican en el valor 1 (valor no deseable) o sea que el proyecto presenta incoherencias sobre el tema de las posibilidades y condiciones de IEA. Para el año 2012 el proyecto de grado muestra una situación diferente en relación a los años 2009, 2010 y 2011, ya que se ubica en el valor 5 (deseable), se supone que tiene una visión articulada, integral y se hace de una manera fundamentada el tema sobre las posibilidades y condiciones de IEA

Categoría C: Tratamiento de los problemas ambientales. 
Bio - grafia. Escritos sobre la Biología y su Enseñanza. ISSN 2027

Edición Extraordinaria. p.p. 344 - 354

Memorias del VIII Encuentro Nacional de Experiencias en Enseñanza de la Biología y la Educación Ambiental. III Congreso Nacional de Investigación en Enseñanza de la Biología.

\begin{tabular}{|c|c|c|c|c|}
\cline { 3 - 4 } \multicolumn{2}{c|}{} & \multicolumn{2}{c|}{ Subcategorías } & \multicolumn{1}{c|}{} \\
\hline Proyectos & Años & C.1. & C.2. & $\begin{array}{c}\text { Valor de la Moda por } \\
\text { subcategoría }\end{array}$ \\
\hline P.36. & $(2009)$ & 1 & 1 & 1 \\
\hline P.15. & $(2010)$ & 1 & 5 & No hay Moda \\
\hline P.6 & $(2011)$ & 1 & 1 & 1 \\
\hline P.7. & $(2012)$ & 5 & 5 & $5^{\star}$ \\
\hline \multicolumn{2}{|c|}{ Valor de la Moda por años } & 1 & 1 y 5 & 1 y 5 \\
\hline
\end{tabular}

Cuadro 5: proyectos de grado de BYEA que consideran el tratamiento de los problemas ambientales

En la categoría C,A la vista de los resultados según el (cuadro 5), en los proyectos de grado de BYEA se observa que el valor predominante es el 1 (valor no deseable) para los años 2009, 2010 y 2011 o sea que se observa en los proyectos incoherencias sobre el tratamiento de los problemas ambientales. Para los años 2010 y 2012 se muestra una situación diferente ya que tiene valores con tendencia en el valor 5 (deseable), se supone que los proyectos tienen una visión articulada, integral y fundamentada sobre el tratamiento de los problemas ambientales, en las subcategorías C.2 (2010) y subcategorías C.1 y C.2 (2012).

\section{CONCLUSIONES}

A pesar que la titulación de Biología y educación Ambiental debería contemplar esta orientación, sólo un $6 \%$ de los proyectos de final de grado contempla la educación ambiental. 
Bio - grafia. Escritos sobre la Biología y su Enseñanza. ISSN 2027

Edición Extraordinaria. p.p. 344 - 354

Memorias del VIII Encuentro Nacional de Experiencias en Enseñanza de la Biología y la Educación Ambiental. III Congreso Nacional de Investigación en Enseñanza de la Biología.

De los proyectos que incorporan la educación ambiental esta se recoge deficientemente ya que tienen una visión aditiva, no se integran sus partes ni se justifican sus relaciones y se hace poco fundamentado el cómo y el qué de la educación ambiental.

La hipótesis de progresión ha permitido detectar las deficiencias del proyecto final de grado, en relación a la incorporación de la educación ambiental en dichos proyectos. Lo cual puede ser una dificultad para que los alumnos la tengan presente en su desempeño profesional.

Sobre el tratamiento de los problemas ambientales, en los proyectos de grado de la licenciatura en BYEA se observa en los proyectos la carencia de incorporar el ámbito educativo en el tratamiento de los problemas ambientales, lo cual supone una visión simplista y tecnocrática. Ello supone una incoherencia con la propia licenciatura en cuyo título aparece la educación ambiental.

\section{BIBLIOGRAFÍA}

Arbat, E. Geli, J, M. (2005). La sostenibilidad en la formación inicial del profesorado: Aplicación del Modelo ACES. Enseñanza de las ciencias. Número extra. VII congreso. Granada España.

Bardin, I. (2002). Análisis de contenido. Ediciones Akal. Madrid.

Junyent, M. (2002). Educación ambiental: un enfocament metodológic en formació inicial del professorat d’Educació Primária. Tesis Doctoral. Universitat de Girona.

Quintana A, y Montgomery W. (2006). Psicología tópicos de actualidad. Lima: UNMSM. Metodología de investigación científica cualitativa.

Wittrock, M.C. (1986). Handbook of research on teaching. New York: Mac Millan Trad cat., La investigación de la enseñanza, I. Enfoques, teorías y métodos. Paidos. Barcelona. 